\title{
On the dimension of an Abelian group
}

\author{
Timo Tossavainen ${ }^{1}$ and Pentti Haukkanen ${ }^{2}$ \\ ${ }^{1}$ Department of Health, Education and Technology, Lulea University of Technology \\ SE-97187 Lulea, Sweden \\ e-mail: timo.tossavainenaltu.se \\ ${ }^{2}$ Faculty of Information Technology and Communication Sciences, Tampere University \\ FI-33014 Tampere University, Finland \\ e-mail: pentti.haukkanen@tuni.fi
}

Received: 13 June 2021

Revised: 17 October 2021

Accepted: 29 October 2021

\begin{abstract}
We introduce a measure of dimensionality of an Abelian group. Our definition of dimension is based on studying perpendicularity relations in an Abelian group. For $G \cong \mathbb{Z}^{n}$, dimension and rank coincide but in general they are different. For example, we show that dimension is sensitive to the overall dimensional structure of a finite or finitely generated Abelian group, whereas rank ignores the torsion subgroup completely.
\end{abstract}

Keywords: Abelian group, Dimension, Perpendicularity.

2020 Mathematics Subject Classification: 11A41, 20K25, 52C99.

\section{Introduction}

We recall that the rank of an Abelian group $G$ is the cardinality of a maximal linearly independent subset of $G$. Further, the fundamental theorem of finitely generated Abelian groups says that every finitely generated Abelian group is isomorphic to a group of the form

$$
G=\mathbb{Z}^{n} \oplus \mathbb{Z}_{a_{1}} \oplus \cdots \oplus \mathbb{Z}_{a_{k}}
$$

The numbers $a_{1}, \ldots, a_{k}$ in the representation of the torsion subgroup $\mathbb{Z}_{a_{1}} \oplus \cdots \oplus \mathbb{Z}_{a_{k}}$ are powers of (not necessarily distinct) primes. Clearly, for such $G$, rank is $n \geq 0$. In particular, a finite Abelian group splits into a direct sum of primary cyclic groups and has rank zero. On the other hand, for each positive integer $n$, there exists a torsion-free Abelian group of rank $n$ that cannot be 
decomposed into a direct sum. For example, $G=(\mathbb{Q},+)$ has rank one but it is indecomposable. So, for finitely generated Abelian groups, rank is a measure of dimensionality that is easy to interpret, but this interpretation does not apply to all Abelian groups.

In addition to rank, there are more advanced tools to study the dimensionality of groups of different types. For example, the minimal dimension of a finite group $G$ is defined as the minimal size of a maximal irredundant set of maximal subgroups of $G[1,3]$. In this article, we show that a useful measure of dimensionality of an Abelian group can be defined also in a more elementary way. It is based on a perpendicularity relation which translates the condition of two vectors $x$ and $y$ being perpendicular to each other in an inner product space into a similar condition for elements of an Abelian group. An advantage of this approach is that it applies to all Abelian groups and is easy to interpret unambiguously. Further, unlike rank, it is sensitive enough to separate finite Abelian groups into more than one category.

The purpose of this article is to provide an introduction to the basic properties of the dimension of an Abelian group which is, as already said, a new concept. Orthogonality relations in an Abelian group have, however, been studied axiomatically already in the 1970s by Davis [2]. His approach is based on studying a disjointness relation, introduced first by Veksler [8] in a linear space, and differs to some degree from the definition to be discussed below. More precisely, he defines an orthogonality relation with aid of four axioms (a pre-orthogonality relation) and a condition on the pre-orthogonal components of singletons (i.e., a set with exactly one element).

A more elementary axiomatisation of perpendicularity, designed merely for educational purposes and compatible with the Euclidean plane, has been studied by Haukkanen, Merikoski, and Tossavainen [5,7].

\section{Perpendicularity}

Let $G=(G,+)$ be an Abelian group and $\perp$ a binary relation in $G$ satisfying

(A1) $\forall a \in G: \exists b \in G: a \perp b$,

(A2) $\forall a \in G \backslash\{0\}: a \not \perp a$,

(A3) $\forall a, b \in G: a \perp b \Rightarrow b \perp a$,

(A4) $\forall a, b, c \in G: a \perp b \wedge a \perp c \Rightarrow a \perp(b+c)$,

(A5) $\forall a, b \in G: a \perp b \Rightarrow a \perp-b$.

We call $\perp$ a perpendicularity in $G$. This definition originated from [4]. It is easy to see that A1-A5 follow immediately from the basic properties of an inner product whenever $\langle a, b\rangle=\langle a, c\rangle=0$. The above definition is meaningful also for non-Abelian groups, but the Abelian groups provide a more natural context for the definition. Indeed the fourth axiom seems more justified if $b+c=c+b$ for all $b, c \in G$, yet $a \perp b \wedge a \perp c$ alone implies both $a \perp(b+c)$ and $a \perp(c+b)$. 
The trivial perpendicularity

$$
x \perp_{0} y \Longleftrightarrow x=0 \vee y=0
$$

exists for every $G$. We call $\perp$ maximal if it is not a subrelation (i.e., a subset) of any other perpendicularity in $G$. Also a maximal perpendicularity always exists. Namely, if $\perp_{1} \subseteq \perp_{2} \subseteq$. . are perpendicularities in $G$, then $\cup_{i=1}^{\infty} \perp_{i}$ is also a perpendicularity in $G$ and the claim now follows from Zorn's lemma.

If $\emptyset \neq A \subseteq G$, the $\perp$-complement of $A$ is

$$
A^{\perp}=\{y \in G: y \perp A\},
$$

where $y \perp A$ means that $y \perp x$ for all $x \in A$. Also, $B \perp A$ means that $y \perp A$ for all $y \in B$. Thus $A^{\perp}$ is the maximal set perpendicular to $A$. In particular, $G^{\perp}=\{0\}$ and $\{0\}^{\perp}=G$. We also set $\emptyset^{\perp}=G$.

We complete this section by recording a lemma from [4, Proposition 4].

Lemma 2.1. If $A \subseteq G$, then $A^{\perp}$ is a subgroup of $G$. If $G$ is cyclic, then $A^{\perp}$ is cyclic.

\section{Dimension of an Abelian group}

We define the $\perp$-dimension of $G$ as follows.

$$
\operatorname{dim}_{\perp}(G)=\min \left\{\operatorname{card}(A): A \subseteq G \text { such that } A^{\perp}=\{0\}\right\}
$$

given that the minimum exists. Here $\operatorname{card}(A)$ is the cardinality of $A$. If the minimum does not exist, we set $\operatorname{dim}_{\perp}(G)=\infty$. Note that the family $\left\{A \subseteq G: A^{\perp}=\{0\}\right\}$ is nonempty, since, at least, $G$ is a member of it. Further, let $\Phi$ be the collection of all maximal perpendicularities in $G$. As previously noted, this collection is nonempty. Then the dimension of $G$ is

$$
\operatorname{dim}(G)=\min _{\perp \in \Phi}\left\{\operatorname{dim}_{\perp}(G)\right\}
$$

if the minimum exists; if it does not, then $\operatorname{dim}(G)=\infty$. This may be written equivalently as

$$
\operatorname{dim}(G)=\inf _{\perp \in \Phi}\left\{\operatorname{dim}_{\perp}(G)\right\} .
$$

It is easy to see that isomorphic Abelian groups have the same dimension.

Let us look at some concrete examples, starting with the simplest cases.

Example 1. Since the trivial perpendicularity $\perp_{0}$ is the only perpendicularity in $G=\{0\}$ and $\emptyset^{\perp_{0}}=\{0\}$, we have $\operatorname{dim}(G)=0$. If $G \neq\{0\}$, then $\operatorname{dim}_{\perp_{0}}(G)=1$. This follows from the fact that, for $A=\{a\}$, where $a \neq 0$, we have $A^{\perp_{0}}=\{0\}$.

Example 2. The Klein four-group $G=\{0, a, b, c\}$ has exactly three nontrivial perpendicularities. One of them is such that $a$ and $b$ are perpendicular to one another and to 0 , and $c$ only to 0 . The other two perpendicularities are similar to this. Consequently, all of them are maximal. Now $\{c\}^{\perp}=\{0\}$ and, therefore, $\operatorname{dim}(G)=1$. 
Example 3. Further, let $V=(G,+, \cdot,\langle\cdot, \cdot\rangle)$ be an inner product space of dimension $n$. Then the relation

$$
x \perp y \Longleftrightarrow\langle x, y\rangle=0
$$

is a perpendicularity in $G$. Let $A=\left\{x_{1}, \ldots, x_{n}\right\}$ be a basis of $V$. Then $A^{\perp}=\{0\}$. Moreover, if $B \subset G$ contains fewer than $n$ elements, then $B^{\perp} \neq\{0\}$. Hence $\operatorname{dim}_{\perp}(G)=n$. If $V$ is of infinite dimension, a slight modification of the above reasoning yields that $\operatorname{dim}_{\perp}(G)=\infty$.

Example 3 shows that our approach is compatible with the ordinary perpendicularity and dimension of a vector space. From a practical point of view, it is still required that there are tools to determine or, at least, to estimate $\operatorname{dim}_{\perp}(G)$ for a given group.

Theorem 3.1. Let $\perp_{1}$ and $\perp_{2}$ be maximal perpendicularities in $G$. Then

$$
\operatorname{dim}_{\perp_{1}}(G)=\operatorname{dim}_{\perp_{2}}(G) .
$$

Proof. Assume that $\perp_{1}$ and $\perp_{2}$ are maximal perpendicularities in $G$ so that

$$
n=\operatorname{dim}_{\perp_{1}}(G)<\operatorname{dim}_{\perp_{2}}(G) .
$$

Let

$$
A=\left\{x_{1}, \ldots, x_{n}\right\}
$$

such that $A^{\perp_{1}}=\{0\}$. By (1), there is $z \neq 0$ so that $z \in A^{\perp_{2}}$. Further, it follows from Lemma 2.1 that $A^{\perp_{2}}$ is a subgroup of $G$. Let $H$ be the subgroup generated by $z$. Then $H \subset A^{\perp_{2}}$.

Because $z \notin A^{\perp_{1}}$, there is $x_{i}$ such that $x_{i} \not \perp_{1} z$. Let $K$ be the subgroup of $G$ generated by $x_{i}$. Next we show that $H \cap K=\{0\}$. Since $z \in A^{\perp_{2}}$, it holds that $z \perp_{2} x_{i}$. It follows from axioms A3-A5 that $m z \perp_{2} x_{i}$ for all $m \in \mathbb{Z}$. Further, axioms A4 and A5 imply that $m z \perp_{2} n x_{i}$ for all $n \in \mathbb{Z}$. From this and axiom A2 it follows that

$$
m z=n x_{i} \Longleftrightarrow m z=n x_{i}=0 .
$$

Define

$$
x \perp y \Longleftrightarrow\left(x \perp_{1} y\right) \vee(x \in H \wedge y \in K) \vee(x \in K \wedge y \in H) .
$$

Now, $x \perp_{1} y$ implies that $x \perp y$. Moreover, $x_{i} \perp z$. Consequently, $\perp_{1}$ is not maximal, which is a contradiction.

In other words, knowing one maximal perpendicularity in $G$ is sufficient for determining the dimension of the group exactly. The next two theorems are useful in studying other cases.

Theorem 3.2. Let $\perp_{1}$ and $\perp_{2}$ be perpendicularities in $G$ such that $\perp_{1} \subseteq \perp_{2}$. Then $\operatorname{dim}_{\perp_{1}}(G) \leq \operatorname{dim}_{\perp_{2}}(G)$.

Proof. It suffices to show that, for an arbirary $A \subseteq G$ such that

$$
A^{\perp_{2}}=\{0\},
$$

we have

$$
A^{\perp_{1}}=\{0\} .
$$

Let $y \perp_{1} A$. Since $\perp_{1} \subseteq \perp_{2}$, we have $y \perp_{2} A$. This and (2) implies that $y=0$. In other words, (3) holds. 
Theorem 3.3. Let $\perp$ be a perpendicularity in $G$ with $\operatorname{dim}_{\perp}(G)=n, n \in \mathbb{N}$. Then

$$
\operatorname{dim}(G) \geq n .
$$

Proof. If $\perp$ is maximal, the claim holds with the equality by Theorem 3.1. If $\perp$ is not maximal, then there is a maximal perpendicularity $\perp_{1}$ containing $\perp$. Then, by Theorem 3.2, $\operatorname{dim}_{\perp_{1}}(G) \geq \operatorname{dim}_{\perp}(G)=n$.

The following example shows that sometimes it is not necessary to find a maximal perpendicularity in order to determine the dimension of an Abelian group.

Example 4. Let $G=(\mathbb{Q} \backslash\{0\}, \cdot)$ and $a=(\operatorname{sgn} a) \prod_{p \in \mathbb{P}} p^{\nu_{p}(a)}$ and $b=(\operatorname{sgn} b) \prod_{p \in \mathbb{P}} p^{\nu_{p}(b)}$. We define $a \perp b$ if and only if $\nu_{p}(a) \neq 0$ implies $\nu_{p}(b)=0$ and $\nu_{p}(b) \neq 0$ implies $\nu_{p}(a)=0$. Let $A \subset \mathbb{Q} \backslash\{0\}$ be finite. Then there is a finite set $P=\left\{p_{1}, p_{2}, \ldots, p_{n}\right\}$ of primes with $p_{n}=\max (P)$ so that every element $a \in A$ can be expressed as

$$
a=(-1)^{\alpha} \prod_{i=1}^{n} p_{i}^{a_{i}}, \quad a_{i} \in \mathbb{Z}, \alpha \in\{0,1\} .
$$

If $p>p_{n}$ is a prime, then $p \in A^{\perp}$. Consequently, $\operatorname{dim}_{\perp}(G)=\infty$. Now, $\operatorname{dim}(G)=\infty$ by Theorem 3.3. It is easy to see that the above perpendicularity is not maximal. In fact, it is contained into the perpendicularity such that $a$ and $b$ are perpendicular if and only if

$$
\sum_{p \in \mathbb{P}} \nu_{p}(a) \nu_{p}(b)=0
$$

\section{Dimension and rank}

For an Abelian group $G=G_{1} \oplus \cdots \oplus G_{n}$, we have

$$
\operatorname{rank}\left(G_{1} \oplus \cdots \oplus G_{n}\right)=\operatorname{rank}\left(G_{1}\right)+\cdots+\operatorname{rank}\left(G_{n}\right) .
$$

A natural question is whether the dimension of an Abelian group has a similar property.

The answer is negative. Above we saw that the dimension of the Klein four-group $K_{4}$ is one. On other hand, this group can be written also as $\mathbb{Z}_{2} \oplus \mathbb{Z}_{2}$, and $\operatorname{dim}\left(\mathbb{Z}_{2}\right)=1$. So,

$$
\operatorname{dim}\left(K_{4}\right) \neq \operatorname{dim}\left(\mathbb{Z}_{2}\right)+\operatorname{dim}\left(\mathbb{Z}_{2}\right) .
$$

The above example also shows that rank and dimension do not always equal each other. However, for quite many groups, they do.

Theorem 4.1. Let $n \geq 2$ and $G \cong \mathbb{Z}^{n}$. Then $\operatorname{dim}(G)=n$.

Proof. Without loss of generality we may assume that $G=\mathbb{Z} \oplus \cdots \oplus \mathbb{Z}$. We write $x=$ $\left(a_{1}, \ldots, a_{n}\right) \in G$ and $y=\left(b_{1}, \ldots, b_{n}\right) \in G$ and define

$$
x \perp y \Longleftrightarrow a_{1} b_{1}+\cdots+a_{n} b_{n}=0 .
$$

We show that $\perp$ is a maximal perpendicularity with $\operatorname{dim}_{\perp}(G)=n$. The latter claim is easier to verify. We write 


$$
\begin{aligned}
\mathbf{e}_{1} & =(1,0, \ldots, 0), \\
\mathbf{e}_{2} & =(0,1, \ldots, 0), \\
& \vdots \\
\mathbf{e}_{n} & =(0,0, \ldots, 1) .
\end{aligned}
$$

Then $\left\{\mathbf{e}_{1}, \ldots, \mathbf{e}_{n}\right\}^{\perp}=\{0\}$. On the other hand, for any $A=\left\{x_{1}, \ldots, x_{n-1}\right\} \subset G$, solving the system of $n-1$ linear equations of type

$$
x_{j 1} b_{1}+x_{j 2} b_{2}+\cdots+x_{j n} b_{n}=0
$$

with respect to $b_{j}$ 's shows that there is $b \neq 0$ such that $b \in A^{\perp}$.

In showing that $\perp$ is maximal, we consider first the case $n=2$. Assume that there is $\perp_{1}$ strictly containing $\perp$. Then there are $a=\left(a_{1}, a_{2}\right)$ and $c=\left(c_{1}, c_{2}\right)$ such that $a \perp_{1} c$ but $a \not \perp c$. Clearly, $a, c \neq 0$. Let $b=\left(b_{1}, b_{2}\right)=\left(a_{2},\left(-a_{1}\right)\right)$; then $a \perp b$. By axioms A2-A5 and $a \perp_{1} c$,

$$
a \neq k c \wedge c \neq k a
$$

for every $k \in \mathbb{Z}$. Further, applying the definition of $\perp$ we see that

$$
b \neq k c \wedge c \neq k b
$$

for every $k \in \mathbb{Z}$ because $a \perp b$ and $a \not \perp c$.

Now, a straightforward calculation shows that

$$
\left\{\begin{array}{l}
a_{1}=b_{1} x+c_{1} y \\
a_{2}=b_{2} x+c_{2} y
\end{array}\right.
$$

holds for

$$
\left\{\begin{array}{l}
x=\left(a_{2} c_{1}-a_{1} c_{2}\right) /\left(b_{2} c_{1}-b_{1} c_{2}\right)=r / q, \\
y=\left(a_{1} b_{2}-a_{2} b_{1}\right) /\left(b_{2} c_{1}-b_{1} c_{2}\right)=s / q .
\end{array}\right.
$$

Observe that (4) and (5) imply that $q, r, s \in \mathbb{Z} \backslash\{0\}$ in (7). Therefore, application of (6) with $x, y$ given in (7) implies

$$
q a=r b+s c .
$$

This leads to a contradiction, because $a \perp_{1} b$ implies that $a \perp_{1} r b$ and $a \perp_{1} c$ implies that $a \perp_{1} s c$, and these together imply that $a \perp_{1} q a$ and, finally, $q a \perp_{1} q a$.

The general case $n \geq 2$ follows in a similar way. First, it is easy to find a set $\left\{b_{1}, \ldots, b_{n-1}\right\}$ for which $a \perp b_{i}$ and $b_{i} \perp b_{j}$ whenever $i \neq j$. Then assuming that there is $\perp_{1}$ containing $\perp$ and that there is an element $c$ such $a \perp_{1} c, a \not \perp c$, and $c \neq k b_{i}$ or $b_{i} \neq k c$, one can write

$$
q a=r_{1} b_{1}+\cdots+r_{n-1} b_{n-1}+r_{n} c
$$

and derive a contradiction with axiom A2.

Theorem 4.2. Let $G \cong \mathbb{Z} \oplus \mathbb{Z} \oplus \cdots$. Then $\operatorname{dim}(G)=\infty$.

Proof. Without loss of generality we may assume that $G=\mathbb{Z} \oplus \mathbb{Z} \oplus \cdots$. We write $x=$ $\left(a_{1}, a_{2}, \ldots\right) \in G$ and $y=\left(b_{1}, b_{2}, \ldots\right) \in G$ and define

$$
x \perp y \Longleftrightarrow a_{1} b_{1}+a_{2} b_{2}+\cdots=0 .
$$


Then $\perp$ is a perpendicularity in $G$. Let $A \subset G$ be a set containing $n, n \geq 2$, elements. Then there is $m \in \mathbb{N}$ such that every $x \in A$ can be written as

$$
x=\left(x_{1}, x_{2}, \ldots, x_{m}, 0,0, \ldots\right) .
$$

Let $y=\left(0,0, \ldots, 0, y_{m+1}, 0,0, \ldots\right)$, where $y_{m+1} \neq 0$. Now, $y \neq 0$ and $y \in A^{\perp}$. Consequently, $\operatorname{dim}_{\perp}(G)>n$. The claim of the theorem follows from Theorem 3.3 since $n$ can be arbitrary large.

Theorem 4.3. Let $G \cong \mathbb{Z}_{m_{1}} \oplus \cdots \oplus \mathbb{Z}_{m_{n}}$, where $\operatorname{gcd}\left(m_{i}, m_{j}\right)=1$ whenever $i \neq j$. Write $x=\left(a_{1}, \ldots, a_{n}\right)$ and $y=\left(b_{1}, \ldots, b_{n}\right)$. Then

$$
x \perp y \Longleftrightarrow \forall i: a_{i}=0 \vee b_{i}=0
$$

is the unique maximal perpendicularity in $G$. Moreover, $\operatorname{dim}(G)=1$.

Proof. The maximality of $\perp$ has been proven in [4, Theorem 14]. Let $a=(1,1, \ldots, 1)$. Then $a \neq 0$ and $\{a\}^{\perp}=\{0\}$.

A reason for the different values of dimension and rank is that the dimension of $G$ describes the 'richness' of the perpendicularity structure throughout $G$. Given a finitely generated Abelian group, rank ignores completely the eventual torsion subgroup in a direct sum, whereas dimension can be remarkably affected by a single finite subgroup of the torsion group. As the following example demonstrates, the existence of a subgroup with a high $\perp$-dimension does not imply that the group itself has a high dimension.

Example 5. Let $\perp$ be the same perpendicularity in $\mathbb{Z}^{n}$ as in the proof of Theorem 4.1 ; then

$$
\operatorname{dim}\left(\mathbb{Z}^{n}\right)=\operatorname{dim}_{\perp}\left(\mathbb{Z}^{n}\right)=n .
$$

An extension of $\perp$ to $G=\mathbb{Z}^{n} \oplus \mathbb{Z}_{2}$ can be constructed as follows. We write

$$
\left(x_{1}, \ldots, x_{n}, x_{n+1}\right)=\left(x, x_{n+1}\right)
$$

and

$$
\left(y_{1}, \ldots, y_{n}, y_{n+1}\right)=\left(y, y_{n+1}\right)
$$

and define

$$
\begin{gathered}
\left(x, x_{n+1}\right) \perp_{1}\left(y, y_{n+1}\right) \Longleftrightarrow\left(x \perp y \wedge\left(x_{n+1}=y_{n+1}=0\right)\right) \vee\left(\left(x, x_{n+1}\right)=0\right) \vee \\
\left(\left(y, y_{n+1}\right)=0\right) .
\end{gathered}
$$

Then $\operatorname{dim}_{\perp_{1}}(G)=1$ because $\{(0, \ldots, 0,1)\}^{\perp_{1}}=\{0\}$.

However, $\perp_{1}$ is not a maximal perpendicularity in $G$. It can be extended further to such a perpendicularity by letting $(0, \ldots, 0,1)$ be perpendicular to every element of $\mathbb{Z}^{n} \oplus\{0\}$ and vice versa. In other words, we define

$$
\begin{aligned}
\left(x, x_{n+1}\right) \perp_{2}\left(y, y_{n+1}\right) \Longleftrightarrow \quad & \left(x \perp y \wedge\left(x_{n+1}=y_{n+1}=0\right)\right) \vee\left(\left(x, x_{n+1}\right)=0\right) \vee \\
& \left(\left(y, y_{n+1}\right)=0\right) \vee\left(x_{n+1}=0 \wedge y=0\right) \vee\left(x=0 \wedge y_{n+1}=0\right) .
\end{aligned}
$$

Also now $\operatorname{dim}_{\perp_{2}}(G)=1$ because $\{(1,1, \ldots, 1)\}^{\perp_{2}}=\{0\}$. Therefore, $\operatorname{dim}_{\perp}(G)=1$. 
Example 1 and Theorem 4.1 above showed that, for every $n \geq 0$, there is a finite or finitely generated Abelian group $G$ with $\operatorname{dim}(G)=n$. Further, Theorem 4.3 showed that the dimension of a finite cyclic group is one. Example 6 below shows that the dimension of a finite group can, however, differ from one. Consequently, it seems that dimension has a higher resolution to detect dimensional differences among finitely generated and even finite Abelian groups than rank has.

Example 6. Let $G=\mathbb{Z}_{3} \oplus \mathbb{Z}_{3}$ and define $\perp$ so that subgroups $G_{1}=\{(0,0),(1,0),(2,0)\}$ and $G_{2}=\{(0,0),(0,1),(0,2)\}$, respectively, and $G_{3}=\{(0,0),(1,1),(2,2)\}$ and $G_{4}=$ $\{(0,0),(1,2),(2,1)\}$, respectively, are perpendicular to each other in both directions. Then, for every $\{a\}$, there is $b \neq 0$ such that $b \in\{a\}^{\perp}$, and $\{(0,1),(1,0)\}^{\perp}=\{(0,0)\}$. Therefore, by Theorem 3.3,

$$
\operatorname{dim}(G) \geq 2
$$

Actually, $\perp$ is also maximal which implies that the dimension of $G$ equals two. To see the maximality of $\perp$, it suffices to notice that, if one takes two nonzero elements $a \in G_{i}$ and $b \in G_{j}$ with $i \neq j$, then every $x \in G$ can be written as $x=m a+n b$, where $m, n \in \mathbb{Z}$. Therefore, a nonzero element $y \in G_{k}, k \notin\{i, j\}$, cannot be perpendicular to $a$ or $b$; otherwise, axioms A4 and A5 would imply that $y$ were perpendicular to itself which violates axiom A2.

It is unknown to us whether, for every $n>2$, there is a finite Abelian group $G$ such that $\operatorname{dim}(G)=n$.

\section{Dimension and homomorphisms}

We conclude this article by recording two theorems. The first one shows that dimension gives a lower bound for the number of cyclic subgroups with trivial pairwise intersections. This theorem may be especially useful for handling the Abelian groups that cannot be decomposed into a direct sum; given such a group $G$ with $\operatorname{dim}(G)=n$, it can, nevertheless, be divided into, at least, $n$ maximally disjoint cyclic subgroups.

Theorem 5.1. Let $G$ be an Abelian group with $\operatorname{dim}(G)=n, n \geq 2$. Then there are cyclic subgroups

$$
G_{1}, \ldots, G_{n} \subset G
$$

where $G_{i} \cap G_{j}=\{0\}$ whenever $i \neq j$.

Proof. Let $\perp$ be a maximal perpendicularity in $G$. We begin by showing that there is a set $A=\left\{x_{1}, \ldots, x_{n}\right\}$ of nonzero elements of $G$ such that $A^{\perp}=\{0\}$ and $x_{i} \perp x_{j}$ whenever $i \neq j$. First, we choose $x_{1} \in G, x_{1} \neq 0$ and set $A_{1}=\left\{x_{1}\right\}$. Then, by Lemma 2.1, $A_{1}^{\perp}$ is a subgroup of $G$ with, at least, one nonzero element $x_{2}$. Indeed, the assumption $\operatorname{dim}(G)=n \geq 2$ implies that $A_{1}^{\perp}$ is nonzero. Now, we have two nonzero elements $x_{1}, x_{2}$ such that $x_{1} \perp x_{2}$.

Assume then that $A_{k}=\left\{x_{1}, \ldots, x_{k}\right\}, 1 \leq k<n$, satisfying $x_{i} \perp x_{j}$ whenever $i \neq j$. Then, again by Lemma 2.1, $A_{k}^{\perp}$ is a subgroup of $G$ with, at least, one nonzero element $x_{k+1}$. This element possesses the property $x_{k+1} \perp A_{k}$. In this way, we finally obtain the set $A=A_{n}$. 
Now, we take $G_{i}=\left\langle x_{i}\right\rangle$, and show that $G_{i} \cap G_{j}=\{0\}$ whenever $i \neq j$. Let $i \neq j$. Since $x_{i} \perp x_{j}$, application of the axioms of perpendicularity shows that $m_{i} x_{i} \perp m_{j} x_{j}$ for all integers $m_{i}, m_{j}$, which implies that the elements $m_{i} x_{i}$ and $m_{j} x_{j}$ are distinct whenever they are nonzero. This proves the claim.

Theorem 5.2. Let $G$ be an Abelian group with $\operatorname{dim}(G)=n$. Let $H$ be an Abelian group and $\phi: G \rightarrow H$ an injective group homomorphism. Then $\operatorname{dim}(\phi(G))=n$.

Proof. The claim follows from the fact that $G$ and $\phi(G)$ are now isomorphic.

Theorem 5.2 is seemingly trivial but it underlines the fact that an $n$-dimensional group can be injectively embedded in an $m$-dimensional group such that $m<n$, cf. Example 5. A similar result does not hold for rank.

\section{Acknowledgements}

We warmly thank the reviewers for carefully reading our manuscript and providing several useful comments.

\section{References}

[1] Burness, T. C., Garonzi, M., \& Lucchini, A. (2020). On the minimal dimension of a finite simple group. Journal of Combinatorial Theory, Series A, 171, Article ID 105175.

[2] Davis, G. (1975). Orthogonality relations on Abelian groups. Journal of the Australian Mathematical Society. Series A, 19, 173-179.

[3] Fernando, R. (2015). On an inequality of dimension-like invariants for finite groups. Preprint, available online at https://arxiv.org/abs/1502.00360.

[4] Haukkanen, P., Mattila, M., Merikoski, J. K., \& Tossavainen, T. (2013). Perpendicularity in an Abelian group. International Journal of Mathematics and Mathematical Sciences, Article ID 983607.

[5] Haukkanen, P., Merikoski, J. K., \& Tossavainen, T. (2011). Axiomatizing perpendicularity and parallelism. Journal of Geometry and Graphics, 15, 129-139.

[6] Mattila, M., Haukkanen, P., Merikoski, J. K., \& Tossavainen, T. (2017). Maximal perpendicularity in certain Abelian groups. Acta Universitatis Sapientiae, Mathematica, 9, 235-247.

[7] Tossavainen, T., \& Haukkanen, P. (2021). Exploring perpendicularity and parallelism through play. To appear in Mathematics Magazine.

[8] Veksler, A. I. (1967). Linear spaces with disjoint elements and their conversion into vector lattices. Leningradskil Gosudarstvennyı Pedagogiceskiı Institut imeni AI Gercena. Ucenye Zapiski, 328, 19-43 (Russian). 